important properties of these NPs. Among the coverage possibilities are organic molecules or metallic layers [9].

Magnetic NPs are beneficial not only because of their relative ease of functionalization, high chemical stability and biocompatibility [12], but also for the detection of molecular targets due to a shift in the surface plasmon resonance (SPR) spectrum [5] (plasmonic sensing); its main advantages lies on its exceptional sensitivity to changes in the dielectric environment [13].

Nowadays there has been a great upswing and extensive use of plasmonic magnetic NPs, mainly in biomedicine like drug delivery [9], due to fast advances and extensive synthesis methods, both in vivo and in situ applications. Plasmonic NPs has the ability to remotely control the spatial position of a NP in real time while tracking its motion provides a new exciting tool for nanoscale sensing. These hot topics are only small examples of the wide range of characteristics that enable their use for biomedical applications.

For the enhancing of many modern technics widely used in real-time feedback for biological mechanisms, plasmonic NPs are a good option to achive it taking into account its magnetic characteristics [9]. Some examples of their use is in the manipulation of cells and proteins [14].

To exploit their novel properties, the studies are focused on their optical properties; since these NPs display a strong optical absorbance when the incident light frequency is in resonance with the collective excitation of the conduction electrons, setting up the SPR condition [15].

In this work, it is described the process of scattering of light by $\mathrm{Au}-\mathrm{Fe}_{3} \mathrm{O}_{4} \mathrm{NPs}$, these were synthesized by co-precipitation method. Towards the characterization of its SP response, it was used a physical model based on Rayleigh scattering and Mie theory for a metal-dielectric interface, solving numerically and compared with experimental UVvisible data.

The aim of this work is provide a specific route to ensure a proper synthesis method of $\mathrm{Au}-\mathrm{Fe}_{3} \mathrm{O}_{4} \mathrm{NPs}$ in order to prepare them for biofunctionalization, avoiding the vast and extensive methods found in literature and huge industrial protocols. The novelty of this work is the analysis of NPs within scattering theory matching the size desired for specific bioapplications and easily tune the optical properties, fitting it for lab-on-a-chip biosensors and analysis in vivo.

\section{Synthesis method}

The most conventional method for obtaining $\mathrm{Fe}_{3} \mathrm{O}_{4} \mathrm{NPs}$ is by coprecipitation [16]. This method consists of mixing ferric and ferrous ions in a 1:2 molar ratio in highly alkaline solutions at room temperature. The size and shape of the iron oxide NPs depends on the type of salt used, the ferric and ferrous ion ratio, the reaction temperature, the $\mathrm{pH}$ solution, etc. Thus, the processing method critically affects the final physical and chemical properties of the iron oxide NPs. In order to avoid the possible oxidation, the synthesis should be done in an anaerobic condition.

The synthesis was made in an aqueous solution without surfactants [17]. Volumes of $1.0 \mathrm{~mL}$ of $1.2 \mathrm{~N} \mathrm{HCl}$ and $12.5 \mathrm{~mL}$ of purified, deoxygenated water (by bubbling nitrogen gas for $30 \mathrm{~min}$ ) were combined, and $2.6 \mathrm{~g}$ of $\mathrm{FeCl}_{3} \cdot 6 \mathrm{H}_{2} \mathrm{O}$ and 1.0 $\mathrm{g}$ of $\mathrm{FeCl}_{2} \cdot 4 \mathrm{H}_{2} \mathrm{O}$ were successively dissolved in the solution while stirring. The resultant solution was added drop wise into $125 \mathrm{~mL}$ of $1.5 \mathrm{M} \mathrm{NaOH}$ solution under vigorous stirring. The last step generated a black precipitate. The magnetism was checked in situ by placing a neodymium magnet near the black precipitate. The precipitate was isolated with the help of a magnetic field, and the supernatant was removed from the precipitate by decantation. Purified deoxygenated water was added to the precipitate two times followed by a methanol wash and finally rinsing with water.

A route to encapsulate the iron oxide NPs is to induce a controlled oxidation of a pure single-metal gold shell. Gold is often employed to passivate the surface of magnetite NPs to avoid oxidation. The most common route employed for preparing the metal functionalized iron oxide is by reduction of $\mathrm{Au}^{3+}$ ions on the surface of the iron oxide; gold coatings provide the stability to the NPs in solution [18-20].

Previously obtained $\mathrm{Fe}_{3} \mathrm{O}_{4}$ were added to a $0.5 \mathrm{mM}$ solution of citric acid and after that it was stirred at $50^{\circ} \mathrm{C}$; surface modification of magnetite by citric acid allows the formation of a stable aqueous dispersion. Then, $4 \mathrm{~g}$ of glucose was dissolved in $40 \mathrm{~mL}$ of citric acid, stabilizing the aqueous solution. Glucose was added to the system as a reducing agent and the mixture was again stirred at room temperature. Finally, reduced $\mathrm{Fe}_{3} \mathrm{O}_{4}$ solution were mixed in a $0.1 \mathrm{M}$ of tetrachloroauric acid aqueous solution $\left(\mathrm{HAuCl}_{4} \cdot 4 \mathrm{H}_{2} \mathrm{O}\right)$ for 30 min using sonication, and then slowly mixed on a stirrer at $50^{\circ} \mathrm{C}$ to allow the adsorption of $\mathrm{Au}^{3+}$ ions onto the magnetite surface. The shell-core NPs was then washed with pure water. A dark purple material was precipitated and separated by centrifugation. The precipitated product was washed with ethanol and it was sonicated for $30 \mathrm{~min}$. The final product can be further separated by centrifugation [18].

\section{Morpho-structural and spectroscopical characterization}

The synthesized NPs exhibit a proper magnetic behaviour since these can be attracted with a neodymium magnet even after the coverage stage with gold. First, it presents 
in Fig. 1 the X-ray diffraction (XRD) pattern of magnetite and $\mathrm{Au}-\mathrm{Fe}_{3} \mathrm{O}_{4} \mathrm{NPs}$ made by an PANalytical X'Pert Powder $\mathrm{X}$-ray diffractometer. The magnetite phase was identified in all synthesized samples by the method previously described. The most intense reflections for this phase are in angle $2 \theta=30.0^{\circ}, 35.5^{\circ}, 43.0,53.5$ and $62.5^{\circ}$, which can be indexed respectively as (220), (311), (400), (422), (511) and (440) planes for a cubic phase, these results are in agreement with previous works [17-19]. The XRD pattern shows that the NPs are highly crystalline. Applying the Scherrer's equation [21] to calculate the crystallite size gives an average value of $19.6 \pm 0.5 \mathrm{~nm}$.

The XRD pattern for $\mathrm{Au}-\mathrm{Fe}_{3} \mathrm{O}_{4}$ exhibits the diffraction peaks for fcc phase of gold and only shows two remaining peaks of $\mathrm{Fe}_{3} \mathrm{O}_{4}$ phase. Data shows diffraction peaks at $2 \theta$ $=38.0^{\circ}, 44.5^{\circ}, 64.5,77.5$ and $81.5^{\circ}$, which can be indexed respectively as (111), (200), (220), (311), and (222) planes for gold in a cubic phase ( $F m 3 m$ space group) [18]. Note that there are two diffraction peaks clearly of magnetite, this is most likely due to the relative heavy atom effect from magnetite. Using Scherrer's equation for calculate the crystallite size of the samples, it obtains a value of $37.5 \pm 0.5 \mathrm{~nm}$.

For scanning electron microscopy (SEM) analysis a few drops of a dilute solution of $\mathrm{Au}-\mathrm{Fe}_{3} \mathrm{O}_{4} \mathrm{NPs}$ were deposited on a silicon substrate, thereafter dried at $80^{\circ} \mathrm{C}$ for $25 \mathrm{~min}$, showing the formation of a thin film. A typical micrograph of this analysis with a Zeiss AURIGA Compact SEM is shown in Fig. 2. Note a strong agglomeration of the particles due to the nature of magnetite [10]. Consider the size, which is observed in the order of $25-40 \mathrm{~nm}$, in agreement with results obtained by XRD analysis.

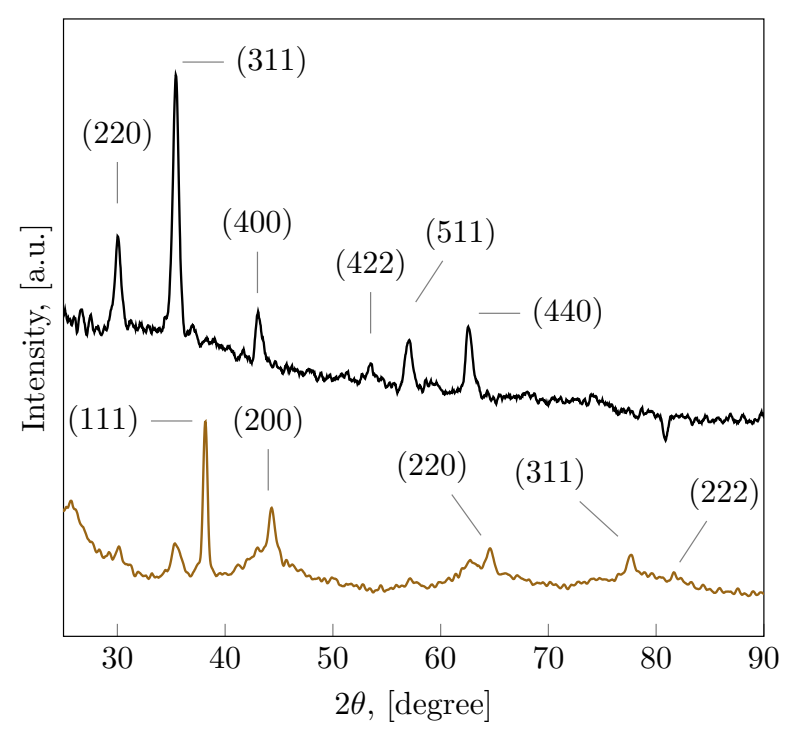

Fig. 1 XRD pattern of magnetite (top) and $\mathrm{Au}-\mathrm{Fe}_{3} \mathrm{O}_{4}$ (bottom) NPs
Samples for scanning transmission electron microscopy (STEM) were prepared on copper grids coated with polymer film in a Jeol JEM-ARM200F STEM witch allows imaging in STEM mode with resolution up to $80 \mathrm{pm}$ with a source of electron field emission of Schottky type. A drop of highly diluted NPs of a sample of $\mathrm{Fe}_{3} \mathrm{O}_{4}$ and another with $\mathrm{Au}-\mathrm{Fe}_{3} \mathrm{O}_{4}$, were previously sonicated by $20 \mathrm{~min}$ at 20 ${ }^{\circ} \mathrm{C}$ and dispersed in ethanol, then it was carefully placed on the copper grid surface and dried at room temperature. Figure 3 shows a representative set of STEM micrographs for magnetite and shell-core NPs. Magnetite are nearly of cubic shape and have an average particle size of $15 \mathrm{~nm}$ while for shell-core an average of $35 \mathrm{~nm}$. Their sizes are in concordance with that obtained in previous works [17-19].

Under the conditions described in the synthesis section, the NPs are expected completely spherical, but after the characterization analysis it can be seen that they appear non-spherical, getting icosahedral shape for shell-core (see Fig. 3c). As is showed by the STEM micrographs, they are highly crystalline. Many of the observed crystalline facets are characteristic of (111) planes for gold. The distance between the nearest neighbor planes is $0.25 \mathrm{~nm}$, which is quite consistent with the calculated $d_{111}$-spacing of lattice planes for gold [20, 22].

Finally, it was established an UV-vis spectroscopy analysis using a Shimadzu UV-Visible 2401 double beam spectrometer. For this technique, it has prepared diluted samples (purified with water) of $\mathrm{Fe}_{3} \mathrm{O}_{4}$ and $\mathrm{Au}-\mathrm{Fe}_{3} \mathrm{O}_{4}$. It presents the results in Fig. 4. Acquired data via UV-vis spectrometer shows a slight peak near $560 \mathrm{~nm}$ corresponding with the particular plasmonic behavior of shell-core NPs [18] and it shows a progressive increase in the absorbance spectrum according the sample

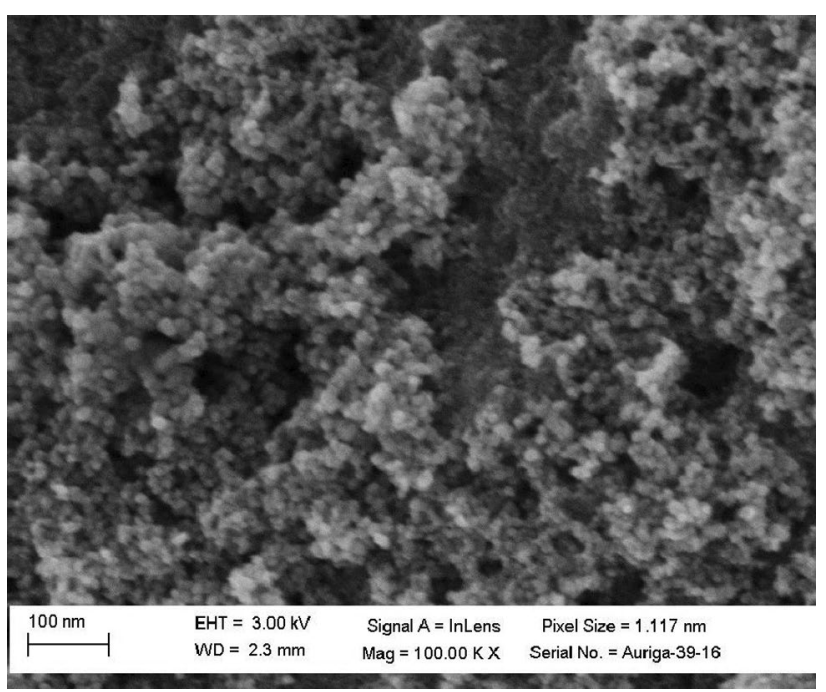

Fig. 2 SEM micrograph of $\mathrm{Au}-\mathrm{Fe}_{3} \mathrm{O}_{4} \mathrm{NPS}$ 

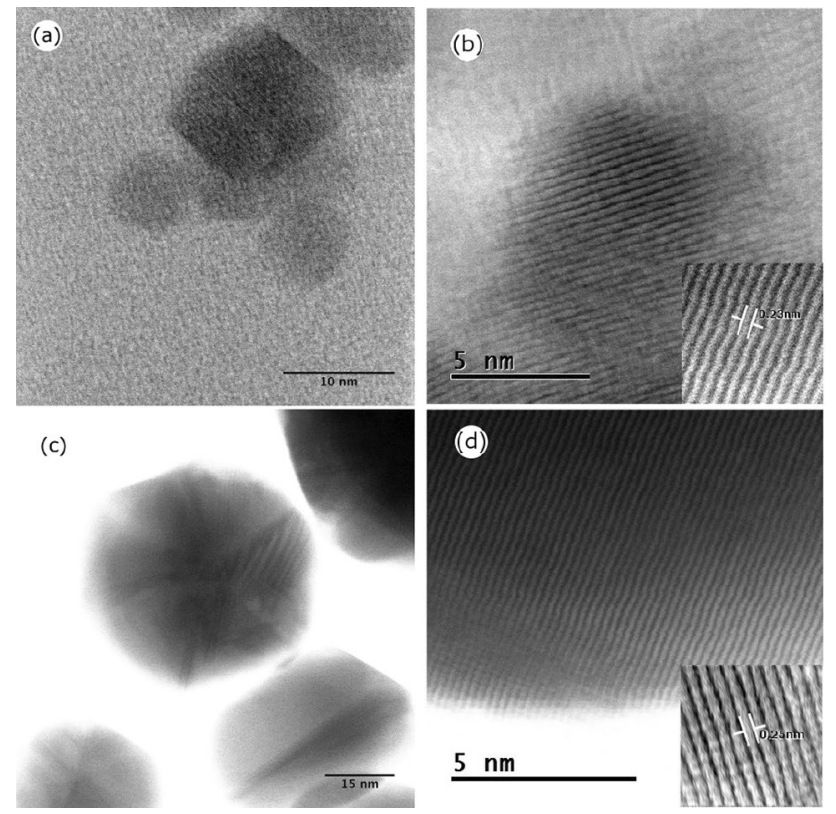

Fig. 3 STEM micrographs of $\mathrm{Fe}_{3} \mathrm{O}_{4}(\mathbf{a}, \mathbf{b})$ and $\mathrm{Au}-\mathrm{Fe}_{3} \mathrm{O}_{4} \mathrm{NPs}(\mathbf{c}, \mathbf{d})$

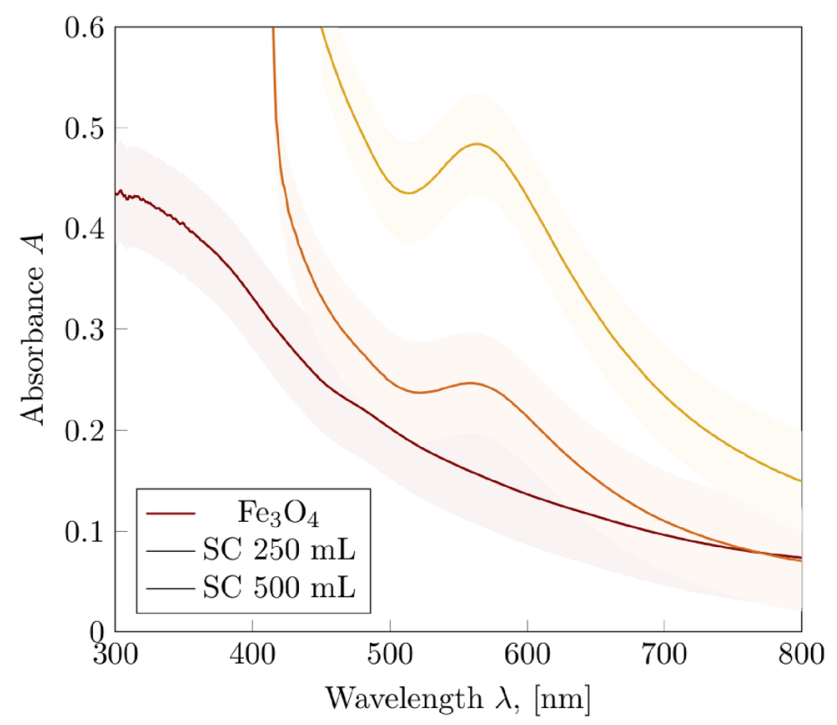

Fig. 4 UV-vis spectra for $\mathrm{Fe}_{3} \mathrm{O}_{4} \mathrm{NPs}$ and shell-core (SC) structure in two different concentrations. Shadow zones corresponds to experimental errors

concentration increase. An important feature in the spectrum is the presence of gold in the system, because magnetite by itself doesn't have a plasmon peak, but gold does.

In order to explain these peaks, it is presented a numerical analysis of the surface plasmon properties based on the electromagnetic and light scattering theories.

\section{Light scattering by nanoparticles}

Light scattering by small particles is the process by which objects such as ice crystals or planetary dust cause observable phenomena like rainbows, the color of the sky, and halos. Maxwell's equations are the basis for its description, and although exact solutions are only known for selected geometries such as spherical and ellipsoidal objects with some assumptions and simplifications, altogether with computational methods are the convenient tools to solve the problem $[23,24]$.

Since the inhomogeneities of the particles are much smaller compared to the wavelength of the incident electromagnetic field, the NPs can be described in terms of a dielectric function which depends on the frequency. It can employ dielectric functions $\epsilon(\omega)$ measured experimentally for metals. These dielectric functions have contributions from interband and intraband electron transitions [1]. It is well established that the optical absorption spectra of alkali and noble metal NPs consist of strong peaks that originate from plasmon excitations $[1,9]$. Therefore, it would also expected that optical spectra of metal nanoshell be plasmonic in origin.

It is considered spherical NPs with a core radius $r_{c}$ and dielectric function $\epsilon_{c^{\prime}}$, the shell has a thickness $r_{\mathrm{t}}-r_{\mathrm{c}}$ and a dielectric function $\epsilon_{s}$, where $r_{\mathrm{t}}$ is the total NPs radius. Embedding medium has a dielectric function $\epsilon_{\mathrm{d}}$.

Rayleigh light scattering theory $[25,26]$ is applicable to particles smaller than the wavelength of incident light. The threshold limit for the maximum particle size that can be modeled by this approximation is $2 \pi r / \lambda \ll 1$, where $r$ is the particle radius (assume spherical) and $\lambda$ is the wavelength of the incident light in the medium of interest. Thereby, and as the synthesized $\mathrm{Au}-\mathrm{Fe}_{3} \mathrm{O}_{4}$ NPs have sizes less than $100 \mathrm{~nm}$ and the gold shell is sufficiently thin, it seems to be proper to apply this approximation and compare the experimental results with theory.

Altogether with Rayleigh approximation, it is presented a complementary calculation focus on the Mie resonances sustained by isolated, noninteracting NPs. As in previous treatment, it is described a spherical NP covered by a thin layered material. It is introduced the very thin monolayer by modifying the usual boundary condition for the magnetic field component tangential to the surface of the sphere, by introducing a finite surface current density at the interface [27].

Commonly, the experiments of light scattering present a characteristic called electromagnetic extinction, due to the attenuation by scattering and absorption of an electromagnetic wave going through the material. It can be suppose only two mechanisms for energy 
loss: scattering $\sigma_{s}$, and absorption $\sigma_{a}$; and their sum will be the extinction cross section $\sigma$, which gives the total energy received by the particle from the incident wave. See in Appendix the equations of extinction cross section for Rayleigh scattering and Mie theory used in this work.

Since extinction cross section is proportional to absorbance $[9,26]$, a comparative with experimental data collected by UV-vis spectroscopy is possible. For this purpose, $\sigma$ will be related with the molar extinction coefficient $\eta$ from Beer-Lambert's Law of spectrophotometry by $A=\eta \ell c$, where for a single wavelength, $A$ is absorbance, $\ell$ is the path length of the sample holder, and $c$ is the sample concentration. For the surrounding aqueous medium it has been used the value of $\epsilon_{\mathrm{d}}=1.77$ corresponding to water. For the shell permittivity $\epsilon_{\mathrm{s}}(\omega)$ it is used the experimentally measured values for gold, after Olmon et al. [28] and Hotta et al. [29]. The value for the dielectric constant $\epsilon_{\mathrm{c}}$ was taken from the analysis of Cuenca et al. [30].

\section{Results and discussion}

Consider first the magnetite NPs which doesn't possesses coverage, assume that for extinction cross section, shell thickness tend to zero $\left(r_{\mathrm{t}}-r_{\mathrm{c}} \simeq 0\right)$ setting only the core radius based on XRD and STEM results.

These results are presented in Fig. 5, note that $\mathrm{Fe}_{3} \mathrm{O}_{4}$ NPs doesn't show an absorbance peak and while the wavelength $\lambda$ increasing, the absorbance is decreasing, exhibiting a monotonically decreasing behaviour which is confirmed by the UV-vis experimental data.

The discrepancy between the model and the UV data is mainly due to the shape of the synthesized magnetite, as well as its agglomeration, which leads to crystals that are not completely spherical, an element that the model assumes. This discrepancy translates into lower absorbance, however, the trend is the same for both results.

Numerical solution of $\sigma$ for $\mathrm{Au}-\mathrm{Fe}_{3} \mathrm{O}_{4} \mathrm{NPs}$ relate extinction cross section under a variation (within the models) of the shell size, assuming a shell thickness that goes from 10 $\mathrm{nm}$ to $20 \mathrm{~nm}$, for which NP size is about $60 \mathrm{~nm}$. It is shown in Fig. 6 the corresponding relative extinction. Clearly, the peak is red-shifted as the radius of NPs is decreased and accordingly it can assume that, if it is required a specific size of NPs, the numerical solutions offer a guide to carefully perform the synthesis, choosing the size of NPs that fit with necessities and thus ensure the proper SPR peak at the desired wavelength.

The values of $r_{\mathrm{c}}$ and $r_{\mathrm{t}}$ can be used to calculate the extinction cross section, showing this result in Fig. 7. It can be seen a peak near $560 \mathrm{~nm}$ from the experimental UV-vis data and a sharp peak in $540 \mathrm{~nm}$ from the

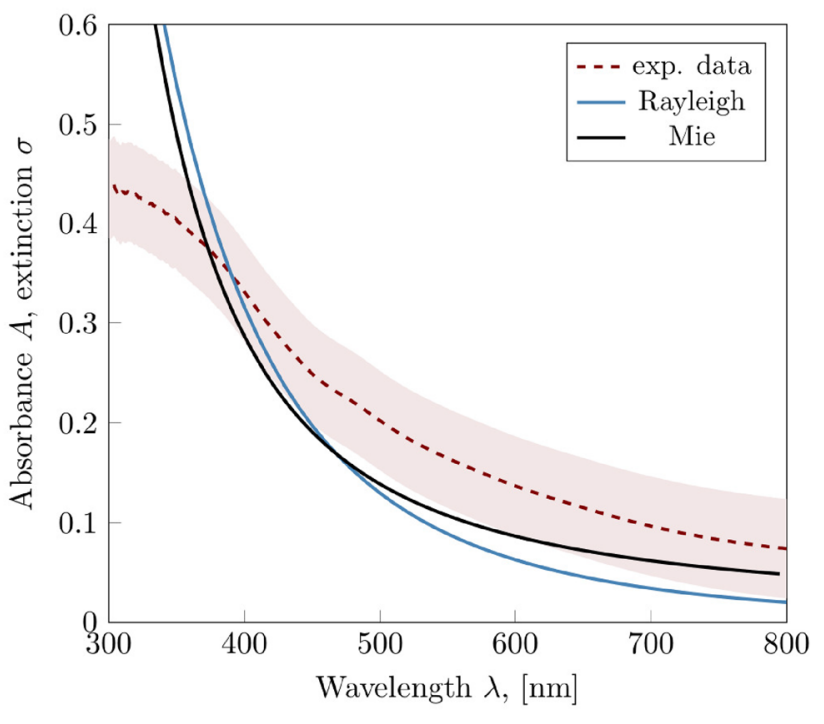

Fig. 5 Numerical solution (solid line) for extinction cross section $\sigma$ of $\mathrm{Fe}_{3} \mathrm{O}_{4} \mathrm{NPs}$ using Rayleigh and Mie theories. Also shown relative absorbance from UV-vis experimental data (dashed line) for comparison, shadow zones corresponds to experimental errors

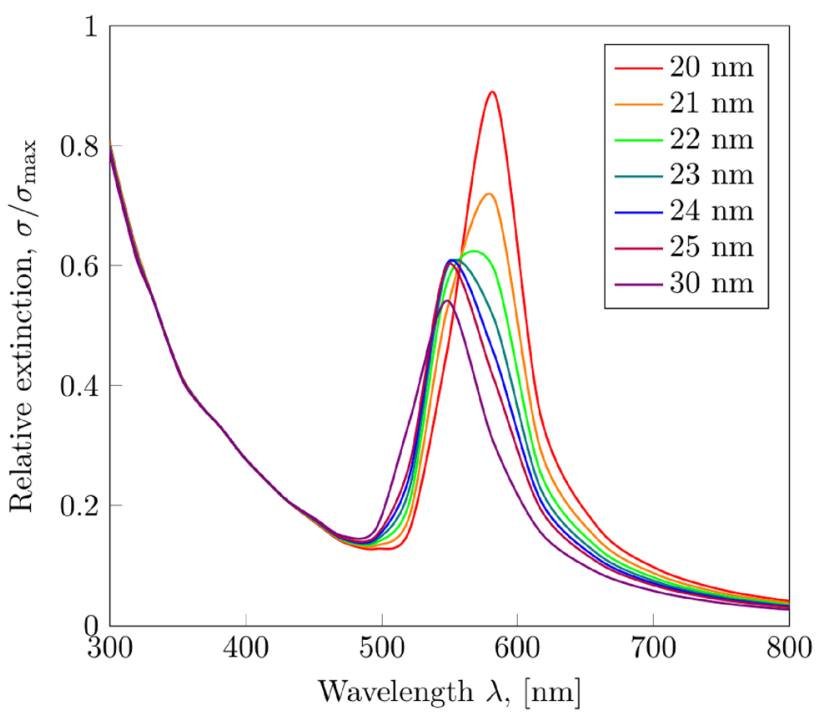

Fig. 6 Relative extinction cross section $\sigma$ for different shell thickness of $\mathrm{Au}-\mathrm{Fe}_{3} \mathrm{O}_{4} \mathrm{NPs}$ (from 20 to $30 \mathrm{~nm}$ ). Its corresponding peaks at different wavelengths are shown. Note the red shift as the NP shell size is decreasing

numerical solution, keeping the sizes previously mentioned. The position of this peak can be attributed to a surface plasmon excitation at this wavelength. The peak from numerical solution compared with that from the experiment, suggest that the most important characteristic from the model is the NP shape, that is, although the fit with experimental data is not complete, it has a similar profile and is evident that some physical properties or 


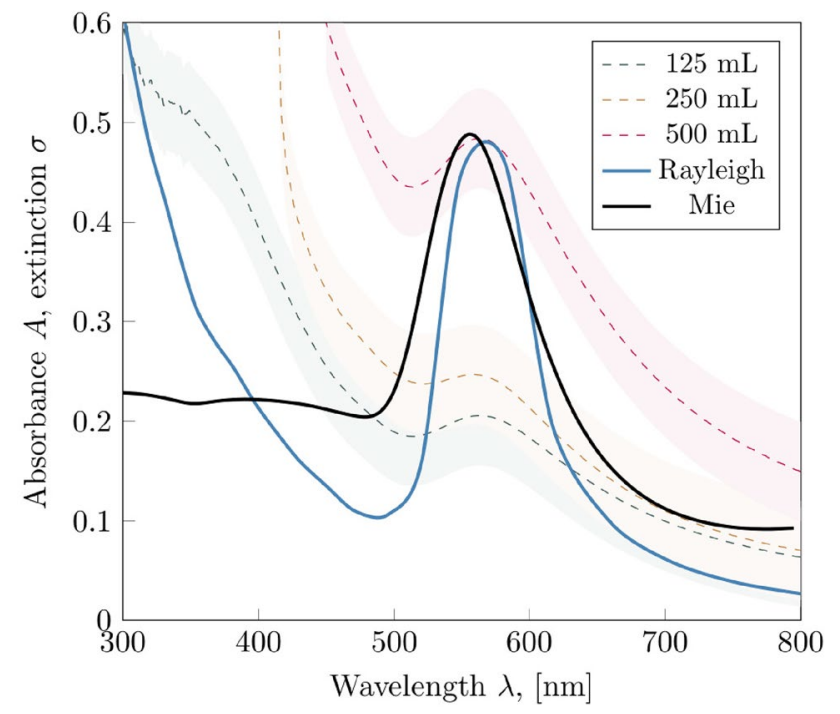

Fig. 7 Numerical solutions of extinction cross section $\sigma$ using light scattering models (Rayleigh and Mie, solid lines) of $\mathrm{Au}-\mathrm{Fe}_{3} \mathrm{O}_{4} \mathrm{NPs}$. Also is presented the relative absorbance from UV-vis experimental results (dashed lines) for three volumes of water and the same amount of NPs $(100 \mu \mathrm{g})$ giving three different concentrations. Shadow zones corresponds to experimental errors. The peak of maximum absorbance is located (experimentally) at $560 \mathrm{~nm}$, while numerical solution with the experimental value of $r_{t}=18.8 \mathrm{~nm}$ gives one located at $580 \mathrm{~nm}$. In addition, is shown the fitted solution taking the same experimental parameters, revealing a NP size of $22 \mathrm{~nm}$. The discrepancies can be attributed to the simplifications of the model and the physical characteristics of the NPs resulting from synthesis not considered in the model, like agglomeration or concentration

characteristics of the NPs have not been considered in the model.

The NP shape was considered spherical for both core and shell, but characterization analysis revealed that NPs synthesis provide a icosahedral like structures (see Fig. 3c) for shell-core and even a different one for magnetite core, being a cube-like structure.

The shift in the position of the peak of absorbance is well correlated with the changes in the dielectric medium. Since gold has a SP behavior [2], there is an enhancement in the plasmonic response after the coated with gold. The surface plasmon response confirmed that there is a correlation between the experimental results and the physical model.

Because the model used to obtain the absorbance considers that the NPs are concentric spheres, a deeper analysis is needed that extensively considers the real shape of the NPs. However, the most important results of this analysis are useful because SP peaks are observed at the wavelengths that are experimentally obtained. And this agrees with the objectives of the work, to be able to synthesize a material with the required characteristics of SP for its later use in biocompatibility applications. An analysis of this material in the form of a very dispersed thin film could help corroborate that the agglomeration is a factor that modifies the absorbance.

\section{Conclusions}

It has been shown that shell-core NPs has a plasmonic shift of absorbance in its visible spectrum. It is very important to control the synthesis process to avoid undesired features is crucial. These synthesized NPs possesses a plasmonic peak and an absorbance profile that fit the expected numerical result.

The average particle size of magnetite NPs was $19.6 \mathrm{~nm}$ as determined by XRD. Same method offers for shell-core NPs reveals an average particle size of $37.5 \mathrm{~nm}$. This was confirmed by SEM, from which one can also see that exhibits some agglomeration. Since gold shows SP behaviour, these NPs have been coated with a gold shell on the surface for enhancing its plasmonic behavior.

It is observed in UV-vis spectroscopy that the peak position of SPR is changed if the size of the shell-core NPs is modified. The extinction cross section which is proportional to the absorbance is well correlated with the experimental results.

One of the most important physical characteristics that will achieve the forthcoming investigations is concerning to plasmonic behavior of shell-core NPs with the specific shape found in these experiments. It will be important to study the changes in the dielectric constant of the surrounding medium in order to ensure functionalized NPs for a different medium like that of the physiological and biomedical assays, for example.

Acknowledgements The author is thankful with CONACyT (Mexico) for graduate grant 403765 and especially wants to thank to $\mathrm{Dr}$ Manuel de Llano for his support and PAPIIT-DGAPA-UNAM (Mexico) research grant IN102417/30. Also wants to thank widely to Dr Goldie Oza for his support and discussions in developing this work.

\section{Compliance with ethical standards}

Conflict of interest The author declare that he has no conflict of interest.

\section{Appendix}

The incident field is time dependent, but it does not vary spatially over the diameter of the metal shell. In this case, the electrostatic solution can be obtained by solving Laplace's equation for the electric potential. The general solution for the potential in each region is of the form [31] 
$\phi_{j}(r, \theta)=\left[A_{j} r+\left(B_{j} / r^{2}\right)\right] \cos \theta$,

where $A_{j}, B_{j}$ ( $j=c, s, d$ for core, shell and medium respectively) are the constants of the monopole and the dipole terms, respectively. The boundary conditions must be specified so that the electric field in the core, shell and embedding medium has continuity in the tangential component as well as continuity in the normal component of the displacement field [32]. In the core region, $B_{c}=0$; in the embedding region, far from the shell, it must recover the potential $\phi_{\mathrm{d}}=-E_{0} r \cos \theta$, thus giving $A_{\mathrm{d}}=-E_{0}$. Application of the boundary conditions to (1) results in a set of four equations and four unknowns that can be solved to obtain the remaining constants. Then, the electric field in each region can be obtained with $E_{j}=-\nabla \phi_{j}(r, \theta)$. The induced field in the region outside the shell is the same as a dipole with an effective dipole moment given by $\mathbf{p}=\epsilon_{\mathrm{d}} \alpha \mathbf{E}$ with $\alpha$ the polarizability, given as [23, 24]:

$\alpha=4 \pi \epsilon_{0} r_{\mathrm{t}}^{3}\left(\frac{\epsilon_{\mathrm{s}} \epsilon_{\mathrm{a}}-\epsilon_{\mathrm{d}} \epsilon_{\mathrm{b}}}{\epsilon_{\mathrm{s}} \epsilon_{\mathrm{a}}+2 \epsilon_{\mathrm{d}} \epsilon_{\mathrm{b}}}\right)$,

where $\epsilon_{0}$ is the permittivity of free space and $\epsilon_{\mathrm{a}}=\epsilon_{\mathrm{c}}(3-2 q)+2 \epsilon_{\mathrm{s}} q, \quad \epsilon_{\mathrm{b}}=\epsilon_{\mathrm{c}} q+\epsilon_{\mathrm{s}}(3-q), \quad$ and $q=1-\left(r_{c} / r_{\mathrm{t}}\right)^{3}$. Then, it can obtain the absorption and the scattering cross sections from the polarizability [23]. For the scattering cross section it obtains

$\sigma_{\mathrm{s}}=\frac{128 \pi^{5}}{3 \lambda^{4}} \epsilon_{\mathrm{d}}^{2} r_{\mathrm{t}}^{6}\left|\frac{\epsilon_{\mathrm{s}} \epsilon_{\mathrm{a}}-\epsilon_{\mathrm{d}} \epsilon_{\mathrm{b}}}{\epsilon_{\mathrm{s}} \epsilon_{\mathrm{a}}+2 \epsilon_{\mathrm{d}} \epsilon_{\mathrm{b}}}\right|^{2}$,

where it has been made for refractive index the approximation $n \simeq \sqrt{\epsilon_{\mathrm{d}}}$ and $\lambda$ is the wavelength of the incident light. Similarly, the absorption cross section is given by:

$\sigma_{\mathrm{a}}=\frac{8 \pi^{2} \sqrt{\epsilon_{\mathrm{d}}}}{\lambda} r_{\mathrm{t}}^{3} \operatorname{Im}\left\{\frac{\epsilon_{\mathrm{s}} \epsilon_{\mathrm{a}}-\epsilon_{\mathrm{d}} \epsilon_{\mathrm{b}}}{\epsilon_{\mathrm{s}} \epsilon_{\mathrm{a}}+2 \epsilon_{\mathrm{d}} \epsilon_{\mathrm{b}}}\right\}$

Finally the extinction cross section is $\sigma=\sigma_{\mathrm{s}}+\sigma_{\mathrm{a}}$.

The Mie theory consider an electromagnetic response of the thin material thus accounted for via a surface conductivity given by

$\sigma_{2 D}(\omega)=-i \omega \epsilon_{0}\left(r_{t}-r_{c}\right)\left[\epsilon_{s}(\omega)-1\right]$.

Within the modified Mie theory [27], the (electric, $a_{\ell}$, and magnetic, $b_{\ell}$ ) Mie scattering coefficients for a spherical particle coated with an arbitrary thin material can be obtained:

$a_{\ell}=\frac{\epsilon_{c} j_{\ell}\left(\rho_{1}\right) \Psi_{\ell}^{\prime}\left(\rho_{2}\right)-\epsilon_{d} j_{\ell}\left(\rho_{2}\right) \Psi_{\ell}^{\prime}\left(\rho_{1}\right)+f_{\sigma}(\omega) \Psi_{\ell}^{\prime}\left(\rho_{1}\right) \Psi_{\ell}^{\prime}\left(\rho_{2}\right)}{\epsilon_{c} j_{\ell}\left(\rho_{1}\right) \xi_{\ell}^{\prime}\left(\rho_{2}\right)-\epsilon_{d} h_{\ell}^{(1)}\left(\rho_{2}\right) \Psi_{\ell}^{\prime}\left(\rho_{1}\right)+f_{\sigma}(\omega) \xi_{\ell}^{\prime}\left(\rho_{2}\right) \Psi_{\ell}^{\prime}\left(\rho_{1}\right)}$ $b_{\ell}=\frac{j_{\ell}\left(\rho_{1}\right) \Psi_{\ell}^{\prime}\left(\rho_{2}\right)-j_{\ell}\left(\rho_{2}\right) \Psi_{\ell}^{\prime}\left(\rho_{1}\right)+f_{\sigma}(\omega) j_{\ell}\left(\rho_{1}\right) j_{\ell}\left(\rho_{2}\right)}{j_{\ell}\left(\rho_{1}\right) \xi_{\ell}^{\prime}\left(\rho_{2}\right)-h_{\ell}^{(1)}\left(\rho_{2}\right) \Psi_{\ell}^{\prime}\left(\rho_{1}\right)+f_{\sigma}(\omega) h_{\ell}^{(1)}\left(\rho_{2}\right) j_{\ell}\left(\rho_{1}\right)}$

where $x_{j}=\left(\omega / c^{\prime}\right) \sqrt{\epsilon_{j}} r_{c}(j=c, d$ mediums $), c^{\prime}$ is the speed of light in vacuum, and it was assumed that the permeabilities of all media are equal to unity. It is introduced the Riccati-Bessel functions $\Psi(\rho)=\rho j_{\ell}(\rho)$ and $\xi(\rho)=\rho h_{\ell}^{(1)}(\rho)$, where $j_{\ell}$ and $h_{\ell}^{(1)}$ are, respectively, the spherical Bessel and Hankel of first kind functions of order $\ell$. Primes denote derivatives with respect to the corresponding function argument. Lastly, defined $f_{\sigma}(\omega)=i \sigma_{2 D}(\omega) /\left(\omega \epsilon_{0} r_{c}\right)$.

Both in the standard Mie theory and in its thin layer variation described here, the extinction cross section is calculated through

$\sigma=\frac{2 \pi}{k_{s}^{2}} \sum_{\ell=1}^{\infty}(2 \ell+1) \operatorname{Re}\left[a_{\ell}+b_{\ell}\right]$

where $k_{s}=\left(\omega / c^{\prime}\right) \epsilon_{s}$ is the wave number in shell medium. Decomposing the cross section into the corresponding electric (magnetic) dipolar, quadrupolar, etc., contributions means retaining in (8) only the $a_{1}, a_{2}$, etc. $\left(b_{1}, b_{2}\right.$, etc.) terms.

\section{References}

1. Noguez C (2007) Surface plasmons on metal nanoparticles: the influence of shape and physical environment. J Phys Chem C 111:3806. https://doi.org/10.1021/jp066539m

2. Halas NJ, Lal S, Chang WS, Link S, Nordlander P (2011) Plasmons in strongly coupled metallic nanostructures. Chem Rev 111:3913. https://doi.org/10.1021/cr200061k

3. Mahmoud MA, Chamanzar M, Adibi A, El-Sayed MA (2012) Effect of the dielectric constant of the surrounding medium and the substrate on the surface plasmon resonance spectrum and sensitivity factors of highly symmetric systems: Silver nanocubes. J Am Chem Soc 134:6434. https://doi.org/10.1021/ja300901e

4. Kho KW, Dinish US, Kumar A, Olivo M (2012) Frequency shifts in SERS for biosensing. ACS Nano 6:4892. https://doi.org/10.1021/ nn300352b

5. Homola J (ed) (2006) Surface plasmon resonance based sensors. Springer, Heidelberg. https://doi.org/10.1007/b100321

6. Pankhurst QA, Connolly J, Jones SK, Dobson J (2003) Applications of magnetic nanoparticles in biomedicine. J Phys D Appl Phys 36:R167. https://doi.org/10.1088/0022-3727/36/13/201

7. Mayergoyz ID (2011) Plasmon resonances in nanoparticle. World Scientific, Singapore. https://doi.org/10.1142/8158

8. Halas NJ (2010) Plasmonics: an emerging field fostered by Nano Letters. Nano Lett 10:3816. https://doi.org/10.1021/nl1032342

9. Lim J, Majetich SA (2013) Composite magnetic-plasmonic nanoparticles for biomedicine: manipulation and imaging. Nano Today 8:98. https://doi.org/10.1016/j.nantod.2012.12.010

10. Wu W, He Q, Jiang C (2008) Magnetic iron oxide nanoparticles: synthesis and surface functionalization strategies. Nanoscale Res Lett 3:397. https://doi.org/10.1007/s11671-008-9174-9

11. Khandhar AP, Ferguson RM, Krishnan KM (2011) Monodispersed magnetite nanoparticles optimized for magnetic fluid 
hyperthermia: implications in biological systems. J Appl Phys 109:07B310. https://doi.org/10.1063/1.3556948

12. Wu W, Wu Z, Yu T, Jiang C, Kim WS (2015) Recent progress on magnetic iron oxide nanoparticles: synthesis, surface functional strategies and biomedical applications. Sci Technol Adv Mater 16:023501. https://doi.org/10.1088/1468-6996/16/2/023501

13. Homola J, Yee SS, Gauglitz G (1999) Surface plasmon resonance sensors: review. Sens Actuators B 54:3. https://doi.org/10.1016/ s0925-4005(98)00321-9

14. Bao J, Chen W, Liu T, Zhu Y, Jin P, Wang L, Liu J, Wei Y, Li Y (2007) Bifunctional $\mathrm{Au}-\mathrm{Fe}_{3} \mathrm{O}_{4}$ nanoparticles for protein separation. ACS Nano 1:293. https://doi.org/10.1021/nn700189h

15. Maier SA (2007) Plasmonics: fundamentals and applications. Springer, New York. https://doi.org/10.1007/0-387-37825-1

16. Alonso-Cristobal $P$, Laurenti $M$, Lopez-Cabarcos $E$, Rubio-Retama $\mathrm{J}$ (2015) Efficient synthesis of core@shell $\mathrm{Fe}_{3} \mathrm{O}_{4} @$ Au nanoparticles. Mater Res Express 2:075002. https://doi.org/10.1088/20531591/2/7/075002

17. Martínez-Mera I, Espinosa-Pesqueira $M$, Pérez-Hernández $R$, Arenas-Alatorre J (2007) Synthesis of magnetite $\left(\mathrm{Fe}_{3} \mathrm{O}_{4}\right)$ nanoparticles without surfactants at room temperature. Mater Lett 61:4447. https://doi.org/10.1016/j.matlet.2007.02.018

18. Wang L, Luo J, Fan Q, Suzuki M, Suzuki IS, Engelhard MH, Lin Y, Kim N, Wang JQ, Zhong CJ (2005) Monodispersed core-shell $\mathrm{Fe}_{3} \mathrm{O}_{4} @$ Au nanoparticles. J Phys Chem B 109:21593. https://doi. org/10.1021/jp0543429

19. Pal S, Morales $M$, Mukherjee $P$, Srikanth $H$ (2009) Synthesis and magnetic properties of gold coated iron oxide nanoparticles. J Appl Phys 105:07B504. https://doi.org/10.1063/1.3059607

20. Sheng Y, Xue J (2012) Synthesis and properties of $\mathrm{Au}-\mathrm{Fe}_{3} \mathrm{O}_{4}$ heterostructured nanoparticles. J Colloid Interface Sci 374:96. https ://doi.org/10.1016/j.jcis.2012.01.034

21. Waseda Y, Matsubara E, Shinoda K (2011) X-ray diffraction crystallography. Springer, Berlin. https://doi.org/10.1007/978-3-64216635-8

22. Yu H, Chen M, Rice PM, Wang SX, White RL, Sun S (2005) Dumbbell-like bifunctional $\mathrm{Au}-\mathrm{Fe}_{3} \mathrm{O}_{4}$ nanoparticles. Nano Lett 5:379. https://doi.org/10.1021/nl047955q
23. van de Hulst HC (2003) Light scattering by small particles. Dover Publications, Dover

24. Mishchenko M, Hovenier J, Travis L (2000) Light scattering by nonspherical particles. Academic Press, London

25. Young AT (1981) Rayleigh scattering. Appl Opt 20:533. https:// doi.org/10.1364/ao.20.000533

26. Averitt RD, Sarkar D, Halas NJ (1997) Plasmon resonance shifts of $\mathrm{Au}-$ Coated $\mathrm{Au}_{2} \mathrm{~S}$ nanoshells: Insight into multicomponent nanoparticle growth. Phys Rev Lett 78:4217. https://doi.org/10.1103/ physrevlett.78.4217

27. Tserkezis C, Gonçalves PAD, Wolff C, Todisco F, Busch K, Mortensen NA (2018) Mie excitons: understanding strong coupling in dielectric nanoparticles. Phys Rev B 98:155439. https:// doi.org/10.1103/physrevb.98.155439

28. Olmon RL, Slovick B, Johnson TW, Shelton D, Oh SH, Boreman GD, Raschke MB (2012) Optical dielectric function of gold. Phys Rev B 86:235147. https://doi.org/10.1103/PhysRevB.86.235147

29. Hotta M, Hayashi M, Nishikata A, Nagata K (2009) Complex permittivity and permeability of $\mathrm{SiO} 2$ and fe3o4 powders in microwave frequency range between 0.2 and $13.5 \mathrm{GHz}$. ISIJ Int 49:1443. https://doi.org/10.2355/isijinternational.49.1443

30. Cuenca JA, Bugler K, Taylor S, Morgan D, Williams P, Bauer J, Porch A (2016) Study of the magnetite to maghemite transition using microwave permittivity and permeability measurements. J Phys: Condens Matter 28:106002. https://doi.org/10.1088/09538984/28/10/106002

31. Griffiths DJ (2017) Introduction to electrodynamics. Cambridge University Press, Cambridge. https://doi.org/10.1017/97811 08333511

32. Haberman R (2019) Applied Partial Differential Equations with Fourier Series and Boundary Value Problems, 5th edn. Pearson, London

Publisher's Note Springer Nature remains neutral with regard to jurisdictional claims in published maps and institutional affiliations. 